\title{
Epidemiology and microbiological characterization of clinical isolates of Staphylococcus aureus in a single healthcare region of the UK, 2015
}

\author{
C. HORNER ${ }^{1 *}$, L. UTSI ${ }^{2}$, L. COOLE ${ }^{2}$ AND M. DENTON ${ }^{1}$ \\ ${ }^{1}$ Regional Public Health Laboratory, National Infection Service, Public Health England, Leeds, UK \\ ${ }^{2}$ Field Epidemiology Service, National Infection Service, Public Health England, Leeds, UK
}

Received 20 May 2016; Final revision 23 September 2016; Accepted 23 September 2016; first published online 28 October 2016

\section{SUMMARY}

We investigated the epidemiology and characterization of isolates of Staphylococcus aureus within the Yorkshire and Humber (YH) region in the UK. In July 2015, each laboratory within YH $(n=14)$ was assigned two consecutive days during which all clinical isolates of $S$. aureus were collected. Isolates were tested for antibiotic susceptibilities and the presence of genes encoding methicillin resistance (mecA and $m e c \mathrm{C}$ ), Panton-Valentine leukocidin (PVL) (lukS-PV), and efflux-mediated chlorhexidine resistance ( $q a c \mathrm{~A})$; isolates were also characterized by spa-types. Minimum inhibitory concentrations (MICs) to chlorhexidine were determined by the broth dilution method. Of 520 isolates collected, 6.2\% were methicillin-resistant S. aureus (MRSA, all mec A-positive) and mupirocin resistance was low [0.8\%, 95\% confidence interval (CI) $0 \cdot 3-2 \cdot 0]$ and only found in MRSA. Carriage of the qacA gene was identified in $1 \cdot 7 \%$ (95\% CI $0 \cdot 8-3 \cdot 3$ ) of isolates and $3 \cdot 5 \%(95 \%$ CI $2 \cdot 2-5 \cdot 4)$ had a chlorhexidine MIC of $4 \mathrm{mg} / \mathrm{l}$. The PVL gene was infrequent $(3 \cdot 7 \%, 95 \%$ CI $2 \cdot 4-5 \cdot 6)$. Genotyping identified 234 spa-types that mapped to 22 clonal complexes. Comparison of these current data with previous work suggest that the widespread use of staphylococcal decolonization regimens over the past decade or more has not had an adverse impact on resistance rates, PVL carriage or the prevalence of specific $S$. aureus lineages.

Key words: Chlorhexidine susceptibility, epidemiology, Panton-Valentine leukocidin.

\section{INTRODUCTION}

Data relating to the antibiotic susceptibilities of Staphylococcus aureus isolates obtained from nonselected patients are lacking. Antibiotic susceptibilities of $S$. aureus are often reported from selected groups, such as cohorts of patients colonized with methicillin-resistant S. aureus (MRSA) or patients with

\footnotetext{
* Author for correspondence: Dr C. Horner, Regional Public Health Laboratory, Public Health England, Leeds LS1 3EX, UK. (Email: Carolyne.horner@phe.gov.uk)

This work was presented at the 26th European Congress of Clinical Microbiology and Infectious Diseases (ECCMID), Amsterdam, 2016 (poster number P0206).
}

healthcare-associated infections. National and international surveillance schemes for these infections generally include $S$. aureus; however, they usually concentrate on the susceptibility of isolates identified from blood cultures or sterile sites [1, 2]. Currently, strains of $S$. aureus are commonly resistant to penicillin due to the production of a penicillinase and resistance to macrolides and quinolones is also widespread [3]. The threat of resistance to glycopeptides, in particular to vancomycin, is a notable concern; however, high-level resistance to vancomycin (MIC $\geqslant 16 \mathrm{mg} / \mathrm{l}$ ) has so far been reported rarely [3].

Bacteraemia caused by $S$. aureus is subject to mandatory surveillance in the UK as part of a national programme to monitor and control healthcare- 
associated infections [4]. System-wide interventions to reduce the number of $S$. aureus bacteraemia cases such as improved line and catheter care, the use of patient isolation and MRSA decolonization have led to substantial declines in the incidence of MRSA cases, although cases of both MRSA and methicillin-susceptible (MSSA) bacteraemia still persist. In the UK, mupirocin and chlorhexidine are the principal antimicrobial agents used for decolonization of patients with $S$. aureus, in particular MRSA, but also to reduce such infections in specific patient populations, such as low birth-weight neonates and those requiring implant surgery [5-7].

Development of mupirocin resistance is an important consideration given its use for decolonization of patients with MRSA and any reduction in the effectiveness of chlorhexidine due to the development of bacterial resistance is of clear public health importance. A recent survey (2015, unpublished local surveillance data) has revealed inconsistency in testing for mupirocin susceptibility across the Yorkshire \& Humber $(\mathrm{YH})$ region of the $\mathrm{UK}$, with half of the laboratories testing mupirocin susceptibility of MRSA isolates only, and no mechanism in place to monitor development of resistance in MSSA strains. Indeed, the prevalence and nature of mupirocin resistance and reduced susceptibility to chlorhexidine in staphylococci has not been systematically monitored in England.

Panton-Valentine leukocidin (PVL) is an extracellular, pore-forming cytotoxin [8] that has been strongly associated with $S$. aureus strains causing skin and soft tissue infections and severe, life-threatening infections, such as necrotizing pneumonia or fasciitis [9]. While any increased incidence of different manifestations of staphylococcal disease may trigger a public health response, staphylococcal bacteraemia and infections known to be associated with PVL-positive strains are subject to enhanced monitoring and initiate local interventions to prevent progression and/or spread of severe disease. National guidance is available for the diagnosis and management of such infections [10], but no such guidance exists for other toxin-related manifestations of staphylococcal disease, such as toxic shock syndrome or staphylococcal enterotoxin disease. Reported rates of PVL-positive strains vary considerably according to the $S$. aureus population tested; from $1.6 \%$ for isolates referred to a reference laboratory for epidemiological typing [11] to $31 \%$ for those tested during a national sentinel surveillance scheme [12]. These numbers are possibly inaccurate estimates of the true burden of PVL, as a large proportion of suspected PVL-associated S. aureus infections are treated based purely on clinical findings without confirmatory testing [13].

Although there has been extensive research into staphylococcal disease and prevention of infection, there remain gaps in key knowledge areas. Currently, an up-to-date description of the antibiotic susceptibilities of clinical $S$. aureus from non-selected populations is lacking and there is uncertainty about the susceptibility of $S$. aureus to the two principal antimicrobials used in standard MRSA decolonization regimens. In addition, the true prevalence of $S$. aureus strains producing PVL is unknown. This study aimed to determine the antibiotic susceptibilities and characteristics of $S$. aureus isolates circulating within the $\mathrm{YH}$ region (population 5.3 million) in particular the prevalence of mupirocin resistance, chlorhexidine nonsusceptibility, and PVL carriage, in order to directly inform public health control strategies and contribute to national knowledge on this topic.

\section{METHODS}

\section{Bacterial isolates}

Clinical isolates of S. aureus were collected over randomly allocated, consecutive 2-day periods throughout July 2015 from the 14 NHS clinical microbiology services laboratories in YH. S. aureus was detected and identified from clinical samples according to the UK Standards for Microbiological Investigations (SMI) document [14] by participating laboratories. The dates of collection did not fall on Mondays, Fridays or weekends in order to minimize intra-site variations in practise. MRSA screening samples and duplicate isolates from the same patient were excluded from collection. Pure colonies of $S$. aureus were transferred onto a nutrient agar slope and transported to a central laboratory for testing. On receipt, the slopes were given a unique study number and stored at $4{ }^{\circ} \mathrm{C}$ until ready to test. Isolates were tested in batches according to procedure. Antibiotic susceptibility testing and DNA extraction for molecular testing were completed first from subcultures of the original isolates from slopes; however, isolates were frozen in glycerol broth for longer term storage and recovered from the freezer for phenotypic chlorhexidine susceptibility.

\section{Data collection}

The following data were collected: source laboratory; laboratory specimen number; date of specimen 
collection; date of $S$. aureus detection; specimen type, whether or not the isolate was from a pure or mixed bacterial culture; the patient's hospital number and National Health Service (NHS) number (or, if missing, patient name); gender; age at specimen collection (or date of birth if age was unavailable) and source of specimen (community or hospital). No clinical data were collected as part of the surveillance, instead a proxy method based on the reported specimen type at time of collection was used to categorize isolates as associated with invasive or non-invasive infection, or colonization.

Isolates were categorized as being associated with an invasive or non-invasive infection based on the reported specimen type at time of collection. Infection was defined as $S$. aureus isolated from an invasive sample type, regardless of whether the culture was pure, or $S$. aureus isolated in pure culture from a non-invasive sample type. Colonization was defined as $S$. aureus isolated from a urine sample collected via a catheter or isolated from a non-invasive sample type in the presence of other bacteria. Specimen source was defined as either hospital (all hospital inpatients) or community (GP surgeries, Accident \& Emergency departments or hospital outpatients).

\section{Antibiotic susceptibility testing}

Susceptibility to a range of antibiotics was tested (benzyl penicillin, chloramphenicol, ciprofloxacin, clindamycin, erythromycin, fusidic acid, nitrofurantoin, gentamicin, linezolid, mupirocin, oxacillin, rifampicin, tetracycline, teicoplanin, trimethoprim, vancomycin) using AST P634 cards and the VITEK $2^{\circledR}$ analyser (bioMérieux, France). A cefoxitin screen for presumptive identification of MRSA and a screen for inducible clindamycin resistance were also included on the AST P634 cards. EUCAST (European Committee on Antimicrobial Susceptibility Testing) breakpoints were used by VITEK 2 for the interpretation of susceptibility results. Isolates identified as resistant to mupirocin by VITEK 2 were tested further with a $200 \mu \mathrm{g}$ mupirocin disc using the EUCAST disc susceptibility method [15].

\section{Chlorhexidine susceptibility testing}

In the absence of an agreed standardized method, chlorhexidine phenotypic susceptibility [minimum inhibitory concentration (MIC) and minimum bactericidal concentration (MBC)] was tested according to methods described by Wand et al. [16]. Briefly, MICs were determined using an overnight tryptone soya broth culture in a broth microdilution method with a concentration range of $0-128 \mathrm{mg} / \mathrm{l}$ chlorhexidine in microtitre plates (Sigma Aldrich, USA). All broth microdilution cultures were incubated at $37^{\circ} \mathrm{C}$ for $\sim 20 \mathrm{~h}$; the MIC was determined by visual inspection against a control and for the MBC, $25 \mu \mathrm{l}$ of non-turbid wells were spotted on tryptic soy agar plates and incubated at $37^{\circ} \mathrm{C}$ for $24 \mathrm{~h}$. Nonsusceptibility to chlorhexidine was defined as MIC $\geqslant 4 \mathrm{mg} / \mathrm{l}[17]$.

\section{Molecular methods}

DNA was extracted from isolates using the QIAxtractor ${ }^{\mathrm{TM}}$ automated column extraction platform (Qiagen, UK) according to the manufacturer's instructions. The presence of genes encoding methicillin resistance genes (mecA and $m e c \mathrm{C}), \mathrm{PVL}(l u k \mathrm{~S}-\mathrm{PV})$, and the species-specific nuc gene were detected simultaneously using a real-time quadruplex PCR assay [18]. The following strains were used as positive controls: ATCC 25923 (lukS-PV-positive), NCTC 10442 (mecA-positive), and a $S$. aureus strain known to be positive for the mec $\mathrm{C}$ gene (provided by Professor A. Kearns, Staphylococcus Reference Unit, London). The presence of the efflux-mediated chlorhexidine resistance gene $(q a c \mathrm{~A})$ was determined by the method of Otter et al. [19] with ST22C64.2 as a positive control. Isolates were genotyped using spa-typing [20] and spa-types were assigned with Ridom StaphType software [21]. S. aureus ATCC 25923 was used as a spa-typing control (t021). MLST-CC were assigned by reference to spa server (http://spa.ridom.de/mlst. shtml) and MLST (http://saureus.mlst.net) databases and assignment was completed by Professor A. Kearns, Staphylococcus Reference Unit, Public Health England, London.

\section{Statistical analysis and power calculations}

The optimal sample size required was calculated for a $0.8 \%$ prevalence of high-level mupirocin resistance (2008, unpublished local surveillance data) with a $95 \%$ confidence interval (CI) of \pm 0.7 precision as 622 isolates. A 2-day sample collection period was expected to yield $\sim 700 \mathrm{~S}$. aureus isolates in $\mathrm{YH}$ which would allow for precise estimates of the prevalence of PVL toxin-positivity and mupirocin and 
chlorhexidine resistance. The prevalence of the foregoing characteristics in addition to the qac A gene were calculated as proportions with associated 95\% Wilson binomial CIs. Prevalence estimates for each primary outcome were presented separately for the following categories: invasive/non-invasive infection, methicillin sensitivity, community/hospital case, and MLST CC. Differences between subgroups were estimated by calculating prevalence ratios (PRs) with corresponding 95\% CIs and tested using Pearson's $\chi^{2}$. PRs for categorical variables (MLST CC) were calculated by pairwise comparisons of each subgroup against the other subgroups combined. All statistical analyses were performed in Stata v. 13.1 (StataCorp, USA).

\section{RESULTS}

\section{Bacterial isolates}

During July 2015, 547 S. aureus isolates were collected from the participating laboratories; 27 isolates were excluded from analysis owing to lack of accompanying datasheet $(n=3), S$. aureus not recovered from original slope due to contamination by Gramnegative organism $(n=5)$; Gram-positive organism other than $S$. aureus submitted $(n=6)$; and duplicate isolates received from the same patient $(n=13)$. Thus, 520 clinical isolates were suitable for analysis.

The number of isolates collected by each laboratory during their allocated 2-day period ranged from 9 to 95 , depending on the size and nature of the laboratory. Isolates from hospital inpatients accounted for $23.5 \%$ and community isolates comprised $74.2 \%$ $(57 \cdot 3 \%, 12.5 \%$ and $4.4 \%$ from GP surgeries, hospital outpatients and A\&E locations, respectively). The location of $2 \cdot 3 \%$ isolates was not recorded. Isolates from invasive infections accounted for only $14(2.7 \%)$ of 520 isolates in the study sample, compared with 320 $(61.5 \%)$ from non-invasive infections and 186 $(35 \cdot 8 \%)$ isolates associated with colonization. Accordingly, the most common specimen type was a wound or skin swab $(413,79 \cdot 4 \%)$. Further characteristics of the study population and $S$. aureus isolates are presented in Table 1 .

\section{Antibiotic susceptibility}

The majority of $S$. aureus isolates were nonsusceptible to benzyl penicillin $(75.8 \%) ; 18 \cdot 7 \%$, $18 \cdot 3 \%$ and $14 \cdot 2 \%$ isolates were non-susceptible to
Table 1. Characteristics of the study population and $\mathbf{S}$. aureus isolates

\begin{tabular}{|c|c|c|}
\hline Characteristic & $n$ & $\%$ \\
\hline \multicolumn{3}{|l|}{ Study population } \\
\hline Total & 520 & $100 \cdot 0$ \\
\hline \multicolumn{3}{|l|}{ Age, years } \\
\hline Median & 55 & \\
\hline Range & $0-97$ & \\
\hline \multicolumn{3}{|l|}{ Gender } \\
\hline Male & 267 & $51 \cdot 3$ \\
\hline Female & 253 & $48 \cdot 7$ \\
\hline \multicolumn{3}{|l|}{ Source } \\
\hline General practice & 298 & $57 \cdot 3$ \\
\hline Accident \& Emergency & 23 & $4 \cdot 4$ \\
\hline Hospital outpatient & 65 & $12 \cdot 5$ \\
\hline Hospital inpatient & 122 & $23 \cdot 5$ \\
\hline Unknown & 12 & $2 \cdot 3$ \\
\hline \multicolumn{3}{|l|}{ S. aureus isolates } \\
\hline \multicolumn{3}{|l|}{ Specimen type } \\
\hline Wound or skin swab & 413 & $79 \cdot 4$ \\
\hline Respiratory & 32 & $6 \cdot 2$ \\
\hline Urine & 12 & $2 \cdot 3$ \\
\hline Blood & 9 & $1 \cdot 7$ \\
\hline Tissue & 3 & $0 \cdot 6$ \\
\hline Urinary catheter & 2 & $0 \cdot 4$ \\
\hline Bronchoalveolar lavage & 1 & $0 \cdot 2$ \\
\hline Bone & 1 & $0 \cdot 2$ \\
\hline Other & 47 & $9 \cdot 0$ \\
\hline \multicolumn{3}{|l|}{ Infection type } \\
\hline Invasive infection & 14 & $2 \cdot 7$ \\
\hline Non-Invasive infection & 320 & $61 \cdot 5$ \\
\hline Colonization & 186 & $35 \cdot 8$ \\
\hline \multicolumn{3}{|l|}{ Methicillin sensitivity } \\
\hline MRSA & 32 & $6 \cdot 2$ \\
\hline MSSA & 487 & $93 \cdot 7$ \\
\hline Unknown & 1 & $0 \cdot 2$ \\
\hline Multidrug resistant* & 84 & $16 \cdot 2$ \\
\hline
\end{tabular}

* Multidrug-resistant $=$ non-susceptible to $\geqslant 3$ classes of antibiotic agent.

fusidic acid, erythromycin and clindamycin, respectively. Other uncommon non-susceptibilities were ciprofloxacin $(8 \cdot 5 \%)$, trimethoprim $(5 \cdot 8 \%)$ and tetracycline $(5.8 \%)$, and $<1 \%$ of isolates were non-susceptible to gentamicin $(0 \cdot 8 \%)$, teicoplanin $(0 \cdot 2 \%)$, and rifampicin $(0 \cdot 2 \%)$. All isolates were susceptible to vancomycin, linezolid, daptomycin, and chloramphenicol. Only four mupirocin-resistant isolates were identified $(0 \cdot 8 \%, 95 \%$ CI $0 \cdot 3-2 \cdot 0)$ (Fig. 1), these were multidrug-resistant (MDR) MRSA isolates with high-level (MIC $>256 \mathrm{mg} / \mathrm{l}$ ) resistance to mupirocin.

Eighty-four $(16 \cdot 2 \%)$ isolates were resistant to $\geqslant 3$ classes of antibiotic and classified as MDR. MRSA isolates accounted for $25 \%(n=21)$ of MDR isolates 
and were commonly resistant to ciprofloxacin, erythromycin $(n=20)$, plus fusidic acid $(n=11)$. Of the 63 MSSA isolates that were also MDR, four were susceptible to benzyl penicillin but non-susceptible to erythromycin, tetracycline, trimethoprim $(n=1)$; clindamycin, erythromycin and tetracycline $(n=1)$, or clindamycin, erythromycin and fusidic acid $(n=2)$. The remaining 59 isolates were non-susceptible to benzyl penicillin, and another two antibiotic classes $(n=$ 40) [most commonly plus erythromycin and clindamycin $(n=22)]$, three classes of antibiotics $(n=18)$, and in one MSSA isolate non-susceptibility to five classes of antibiotic agents was recorded. The teicoplaninresistant isolate (MIC $4 \mathrm{mg} / \mathrm{l}$ ) was confirmed by the national reference laboratory and resistance was not due to a single identifiable or transferrable genetic element.

Thirty-two isolates $(6 \cdot 2 \%)$ were positive for the $m e c \mathrm{~A}$ gene and classified as MRSA; no isolate harboured the mec $\mathrm{C}$ gene. One isolate was positive for the mecA gene but susceptible to oxacillin and was excluded from further analysis (labelled as unknown in Table 1). The majority of MRSA isolates were non-susceptible to ciprofloxacin $(81 \cdot 3 \%)$, erythromycin (59.4\%), fusidic acid, (37.5\%) and clindamycin $(34 \cdot 4 \%)$. Hospital inpatient isolates were more than twice as likely to be methicillin-resistant than community isolates $(10 \cdot 7 \% v s .4 \cdot 9 \%$, PR $2 \cdot 2,95 \%$ CI $1 \cdot 1-4 \cdot 2$, $P=0.032$ ); however, there was no significant association between MRSA and invasive or non-invasive sample types.

\section{Chlorhexidine susceptibility}

Of the 519 isolates tested for phenotypic susceptibility to chlorhexidine (one isolate was not recovered from frozen storage), $18(3 \cdot 5 \%, 95 \%$ CI $2 \cdot 2-5 \cdot 4)$ had a MIC equal to $4 \mathrm{mg} / \mathrm{l}$; no isolates exhibited a MIC $>4 \mathrm{mg} / \mathrm{l}$. Nine isolates were positive for the qacA gene $(1 \cdot 7 \%, 95 \%$ CI $0 \cdot 9-3 \cdot 3)$, one of which was also positive for PVL. None of the qacA-positive isolates carried the mecA gene. There was very little correlation between carriage of the qacA gene and phenotypic non-susceptibility to chlorhexidine as only one isolate which possessed the qac $\mathrm{A}$ gene also exhibited a chlorhexidine MIC of $4 \mathrm{mg} / \mathrm{l}$ whereas 27 isolates with or without $q a c \mathrm{~A}$ had a similar MIC (5\%, 95\% CI 3.4-7·2) (Fig. 1).

There were 181 isolates that gave a chlorhexidine $\mathrm{MBC}$ of $>4 \mathrm{mg} / \mathrm{l}$; however, reading the MBC results was not straightforward. For instance, 79 isolates gave variable $\mathrm{MBC}$ values at different dilutions (i.e. no growth at one dilution, growth at the next, no growth at the next, and growth at the final dilution). Such variable growth was attributed to the presence of variant or 'hetero-resistant' populations. Figure 2 shows the frequency distribution of MIC and MBC to chlorhexidine in all clinical $S$. aureus isolates. There were no significant differences in the prevalence of isolates with MIC $4 \mathrm{mg} / \mathrm{l}$ chlorhexidine or qacA-positive isolates between MRSA and MSSA, community and hospital settings, or non-invasive and invasive sample types (Table 2).

\section{Prevalence of PVL-positive $S$. aureus}

Nineteen $(3 \cdot 7 \%, 95 \%$ CI $2 \cdot 4-5 \cdot 6)$ isolates were identified as PVL-positive (Fig. 1). There was no significant difference in prevalence of PVL-positivity between MRSA and MSSA, community and hospital settings, or non-invasive and invasive sample types (Table 2).

\section{Genetic characterization}

Two hundred and thirty-four different spa-types were identified from 514 isolates, mapping to 22 different MLST CCs. Six isolates were spa non-typable, and 22 spa-types were unable to be mapped to MLST $\mathrm{CC}$ without further investigation. Sixteen new spatypes were identified and submitted to RIDOM.

As expected, MSSA isolates belonged to a range of CC ( $n=16$ lineages), compared with MRSA isolates. The majority of isolates $(n=421,81 \%)$ were grouped in one of nine CCs; CC30 (13.5\%), CC15 (12.5\%), and $\mathrm{CC} 1(11 \%)$ being the most predominant (Fig. 3). MRSA isolates fell in six CCs with CC59 and CC22 (EMRSA-15) being the most prevalent ( $n$ $=13$ isolates per CC). All four mupirocin-resistant MRSA isolates belonged to CC59. PVL-positive isolates were distributed in $11 \mathrm{CCs}$ with the highest prevalence in CC8 (10.4\% of isolates; PR 3.45, 95\% CI $1 \cdot 29-9 \cdot 27)$ and $\mathrm{CC} 121 \quad(22 \cdot 2 \%$ of isolates; PR $6 \cdot 53,95 \%$ CI $1 \cdot 75-24 \cdot 3)$ compared to isolates in all other identified CCs. Isolates that carried the qacA gene were distributed among four lineages (CC30, $\mathrm{CC} 5, \mathrm{CC} 8$ and $\mathrm{CC} 88$ ), and the prevalence of qacApositive isolates was significantly higher in CC88 [33.3\% of isolates $(n=2)$; PR $26 \cdot 3,95 \%$ CI $6 \cdot 59-105]$.

\section{DISCUSSION}

These data from a prospective cross-sectional prevalence survey provide a reassuring picture of $S$. aureus 


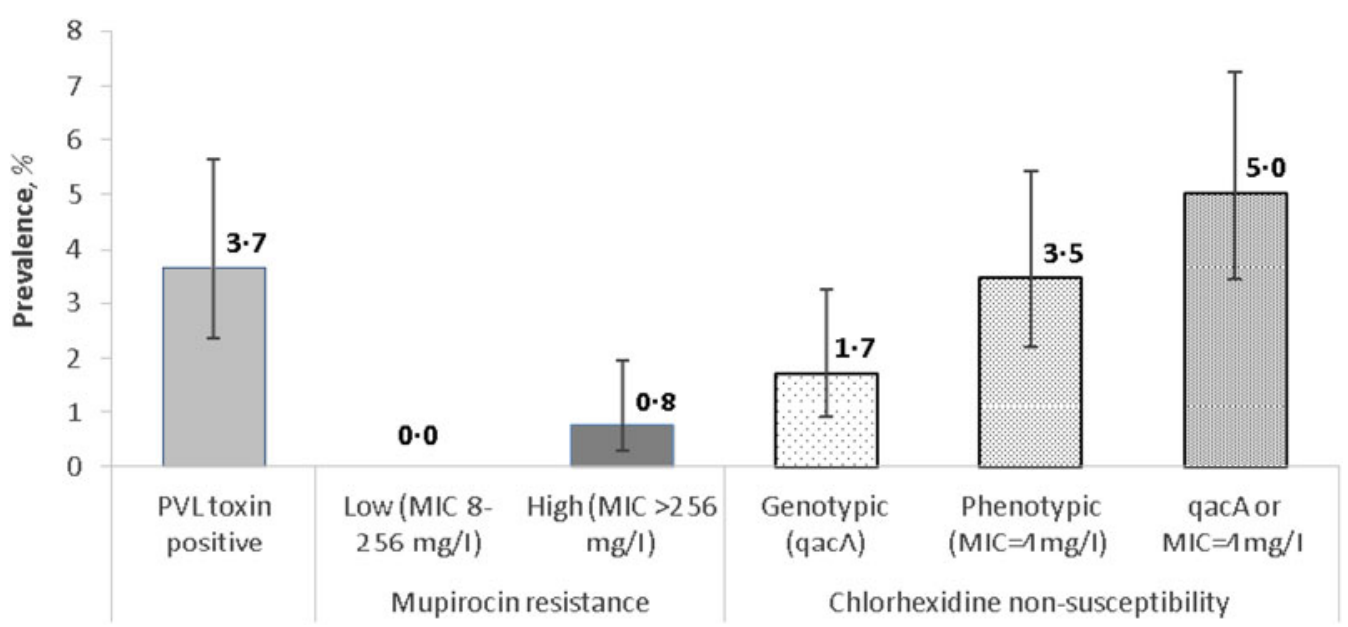

Fig. 1. Prevalence of Panton-Valentine leukocidin (PVL) toxin, mupirocin and chlorhexidine resistance in clinical $S$. aureus isolates $(n=520)$. Error bars represent $95 \%$ Wilson binomial confidence intervals around the prevalence estimate. MIC, Minimum inhibitory concentration.

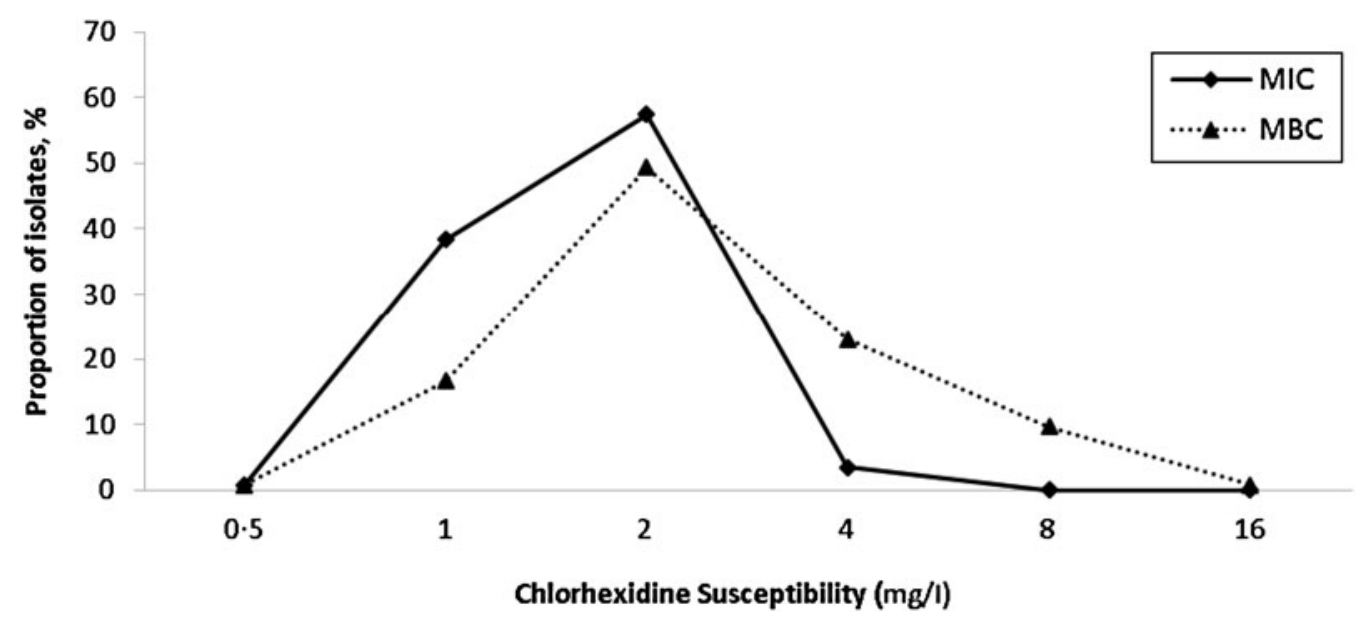

Fig. 2. Distribution of the minimum inhibitory concentrations (MIC) and minimum bactericidal concentrations (MBC) of chlorhexidine for clinical $S$. aureus isolates. Non-susceptibility to chlorhexidine was defined as an MIC $\geqslant 4 \mathrm{mg} / \mathrm{l}$ [17].

within a single healthcare region of the UK at a time when antibiotic resistance is a threat to the treatment of infectious disease.

The prevalence of non-susceptibility in $S$. aureus isolates to various antibiotic agents identified in the present study bears comparison with rates reported by the British Society for Antimicrobial Chemotherapy (BSAC), which summarize the non-susceptibility trends in staphylococci from cases of bacteraemia in the UK and Ireland (2001-2006) [2], and with contemporaneous but non-published data of the antibiotic susceptibilities of $S$. aureus $(n=2640)$ from clinical samples of a non-selected community population in two large UK cities (Leeds and Bradford) during 2010-2012. Of note, the prevalence of non-susceptibility to ciprofloxacin in the present study was similar to local data but lower than that of BSAC; prevalence of non-susceptibility to erythromycin and fusidic acid were higher in the present study compared to previous local data or with BSAC data; whereas prevalence of non-susceptibility to trimethoprim has decreased (Table 3). Nevertheless, isolates defined as resistant to $\geqslant 3$ antibiotic classes remained susceptible to at least four classes of antibiotics tested, leaving options for treatment.

We have identified a low prevalence of MRSA $(6 \cdot 2 \%)$ compared to $11 \%$ noted previously from the earlier local data (2010-2012). As such, flucloxacillin remains a reasonable first-line empirical choice for treatment of non-complicated $S$. aureus infections in 
Table 2. Differences in the prevalence of mupirocin resistance, chlorhexidine non-susceptibility, and PantonValentine leukocidin (PVL) in clinical isolates of MSSA and MRSA*, from community and hospital settings, and from non-invasive and invasive infections*

\begin{tabular}{|c|c|c|c|c|c|c|c|c|c|}
\hline & \multicolumn{6}{|c|}{ Prevalence } & \multirow[b]{3}{*}{ PR } & \multirow[b]{3}{*}{$95 \% \mathrm{CI}$} & \multirow[b]{3}{*}{$P$ value } \\
\hline & \multicolumn{3}{|c|}{ MSSA } & \multicolumn{3}{|c|}{ MRSA } & & & \\
\hline & $N$ & $n$ & $\%$ & $N$ & $n$ & $\%$ & & & \\
\hline Mupirocin resistance & 487 & 0 & $0 \cdot 0$ & 32 & 4 & $12 \cdot 5$ & $133 \cdot 1$ & $(9 \cdot 16-\infty)$ & $<0.001$ \\
\hline \multicolumn{10}{|l|}{ Chlorhexidine non-susceptibility } \\
\hline Phenotypic $(\mathrm{MIC}=4 \mathrm{mg} / \mathrm{l}) \dagger$ & 486 & 15 & $3 \cdot 1$ & 32 & 3 & $9 \cdot 4$ & $3 \cdot 04$ & $(0 \cdot 93-9 \cdot 95)$ & 0.093 \\
\hline$q a c \mathrm{~A}$ or $\mathrm{MIC}=4 \mathrm{mg} / \mathrm{l}$ & 486 & 23 & $4 \cdot 7$ & 32 & 3 & $9 \cdot 4$ & 1.98 & $(0 \cdot 63-6 \cdot 25)$ & $0 \cdot 212$ \\
\hline \multirow[t]{4}{*}{ PVL toxin positivity } & 487 & 17 & $3 \cdot 5$ & 32 & 2 & $6 \cdot 3$ & 1.79 & $(0 \cdot 43-7 \cdot 41)$ & $0 \cdot 330$ \\
\hline & \multicolumn{6}{|c|}{ Prevalence } & \multirow[b]{3}{*}{ PR } & \multirow[b]{3}{*}{$95 \% \mathrm{CI}$} & \multirow[b]{3}{*}{$P$ value } \\
\hline & \multicolumn{3}{|c|}{ Community } & \multicolumn{3}{|c|}{ Hospital } & & & \\
\hline & $N$ & $n$ & $\%$ & $N$ & $n$ & $\%$ & & & \\
\hline \multicolumn{8}{|l|}{ Chlorhexidine non-susceptibility } & $(1 \cdot 00-90 \cdot 4)$ & $0 \cdot 045$ \\
\hline Genotypic (qacA) & 386 & 6 & $1 \cdot 6$ & 122 & 3 & $2 \cdot 5$ & $1 \cdot 58$ & $(0 \cdot 40-6 \cdot 23)$ & $0 \cdot 454$ \\
\hline Phenotypic $(\mathrm{MIC}=4 \mathrm{mg} / \mathrm{l}) \dagger$ & 385 & 12 & $3 \cdot 1$ & 122 & 6 & 4.9 & $1 \cdot 58$ & $(0 \cdot 61-4 \cdot 12)$ & $0 \cdot 399$ \\
\hline$q a c \mathrm{~A}$ or $\mathrm{MIC}=4 \mathrm{mg} / \mathrm{l}$ & 385 & 17 & $4 \cdot 4$ & 122 & 9 & $7 \cdot 4$ & $1 \cdot 67$ & $(0 \cdot 76-3 \cdot 65)$ & $0 \cdot 237$ \\
\hline \multirow[t]{4}{*}{ PVL toxin positivity } & 386 & 11 & $2 \cdot 8$ & 122 & 8 & $6 \cdot 6$ & $2 \cdot 30$ & $(0 \cdot 95-5 \cdot 60)$ & $0 \cdot 095$ \\
\hline & \multicolumn{6}{|c|}{ Prevalence } & \multirow[b]{3}{*}{ PR } & & \\
\hline & \multicolumn{3}{|c|}{ Non-invasive } & \multicolumn{3}{|c|}{ Invasive } & & \multirow[b]{2}{*}{$95 \% \mathrm{CI}$} & \multirow[b]{2}{*}{$P$ value } \\
\hline & $N$ & $n$ & $\%$ & $N$ & $n$ & $\%$ & & & \\
\hline Mupirocin resistance & 320 & 3 & $0 \cdot 9$ & 14 & 0 & $0 \cdot 0$ & $3 \cdot 06$ & $(0 \cdot 00-47 \cdot 2)$ & $0 \cdot 168$ \\
\hline \multicolumn{10}{|l|}{ Chlorhexidine non-susceptibility } \\
\hline Genotypic (qacA) & 320 & 5 & $1 \cdot 6$ & 14 & 0 & $0 \cdot 0$ & 1.95 & $(0 \cdot 00-25 \cdot 3)$ & $0 \cdot 241$ \\
\hline Phenotypic $(\mathrm{MIC}=4 \mathrm{mg} / \mathrm{l}) \dagger$ & 319 & 8 & $2 \cdot 5$ & 14 & 0 & $0 \cdot 0$ & $1 \cdot 26$ & $(0 \cdot 00-14 \cdot 5)$ & $0 \cdot 341$ \\
\hline$q a c \mathrm{~A}$ or $\mathrm{MIC}=4 \mathrm{mg} / \mathrm{l}$ & 319 & 12 & $3 \cdot 8$ & 14 & 0 & $0 \cdot 0$ & $0 \cdot 85$ & $(0 \cdot 00-9 \cdot 11)$ & $0 \cdot 455$ \\
\hline PVL toxin positivity & 320 & 14 & $4 \cdot 4$ & 14 & 1 & $7 \cdot 1$ & $1 \cdot 63$ & $(0 \cdot 23-11 \cdot 6)$ & $0 \cdot 481$ \\
\hline
\end{tabular}

MIC, Minimum inhibitory concentration; PR, prevalence ratio; CI, confidence interval.

$P$ value $=$ Fisher's exact test $\mathrm{p}$ value; statistically significant prevalence ratios are presented in bold type.

* Prevalence ratios and confidence intervals have been estimated for categories with zero cell counts using a Woolf-Haldane correction.

$\dagger$ One isolate was not recovered from frozen storage.

this geographical area. It is important, however, that GPs remain aware of the prevalence of MRSA circulating in the local community so that patients with previous history or risk factors for MRSA colonization/infection can be treated accordingly. It is reassuring that isolates carrying the $m e c \mathrm{C}$ gene were not identified during this surveillance. $m e c \mathrm{C}$, previously known as $m e c \mathrm{~A}_{\mathrm{L} 251}$, was first reported in 2011, initially from dairy cattle but subsequently identified in clinical samples from humans [22]. The prevalence of
mecC-positive MRSA remains low in England and throughout most of Europe [23, 24]; however, with an increased prevalence in Denmark [25] vigilance is recommended for widespread emergence of $S$. aureus carrying this gene.

Despite the widespread use of mupirocin and chlorhexidine in MRSA decolonization, non-susceptibility to these agents remains low. Although the emergence of resistance to mupirocin with increasing use has been documented in centres in Brazil [26] and 


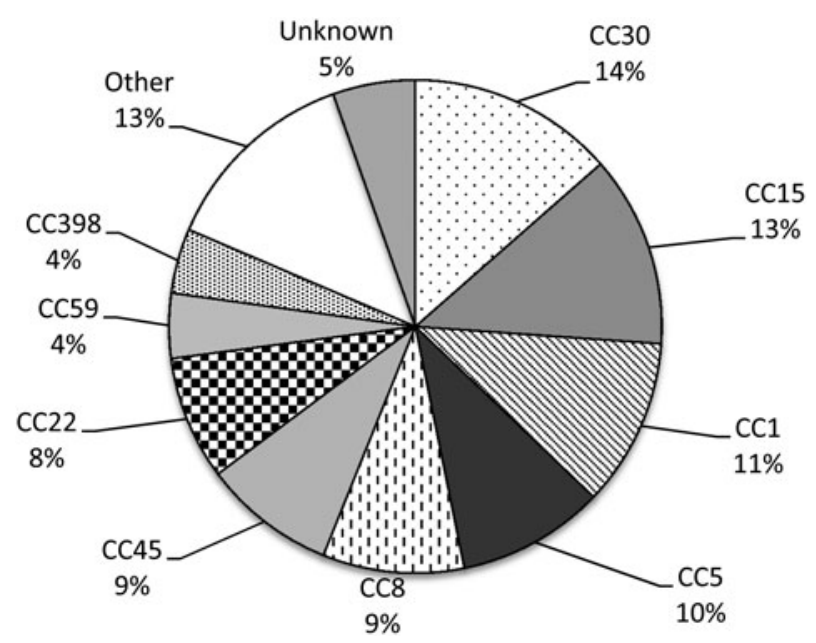

Fig. 3. Most commonly occurring multilocus sequence type clonal complexes (CCs) of clinical $S$. aureus isolates $(n=520)$. Other comprise: CC7 $(n=12), \operatorname{CC} 25 \quad(n=12)$, CC121 $(n=9), \operatorname{CC} 97(n=8), \operatorname{CC} 80(n=6), \operatorname{CC} 88(n=6)$, CC12 $(n=5), \operatorname{CC} 9(n=3), \operatorname{CC} 152(n=2), \operatorname{CC} 182(n=2)$, CC101 $(n=1), \operatorname{CC} 1290(n=1), \operatorname{CC} 20(n=1)$; unknown comprise: no CC match $(n=22)$, no spa-type data $(n=6)$.

Canada [27], other studies have not demonstrated such an association. Low levels of mupirocin resistance over a 10-year period have been identified in Belgium; of 1971 isolates tested, 39 MRSA (3.1\%) and four MSSA (0.6\%) isolates showed high-level mupirocin resistance $(>256 \mathrm{mg} / \mathrm{l})$ [28]. A 4-year repeated point-prevalence study between 1999 and 2003 in the surgical units of one of the major teaching hospitals in the $\mathrm{YH}$ region found no evidence for the sustained emergence of mupirocin resistance after the introduction of a mupirocin-based peri-operative prophylaxis regimen for the prevention of MRSA [29]. Over a decade later, our study has shown that the prevalence of mupirocin resistance in $S$. aureus remains low, both in the same teaching hospital and across other locations within the region. Interestingly, the only mupirocin-resistant isolates $(n=4)$ identified here belonged to CC59, a lineage that was associated with mupirocin resistance and whose prevalence increased during a 4-year period of surveillance of MRSA in care homes for the elderly in the $\mathrm{YH}$ region [30]. The four mupirocin-resistant isolates were submitted by four different laboratories and were not associated with PVL carriage.

The low prevalence of PVL-positive $S$. aureus reported here does not appear to indicate a large, undetected pool of such strains circulating in our region. A similarly low prevalence of PVL-positive isolates $(1 \cdot 6 \%, 95 \%$ CI $0 \cdot 7-3 \cdot 0)$ was found in $515 \mathrm{~S}$. aureus
Table 3. Comparison of the prevalence of nonsusceptibility of S. aureus recorded by British Society for Antimicrobial Chemotherapy (BSAC) surveillance data (2001-2006), non-published local surveillance data (2010-2012) and the present study (July 2016)

\begin{tabular}{|c|c|c|c|c|}
\hline $\begin{array}{l}\text { Antibiotic } \\
\text { agent }\end{array}$ & $\begin{array}{l}S . \\
\text { aureus }^{*}\end{array}$ & $\begin{array}{l}\text { BSAC } \\
(2001- \\
2006) \\
(\%)\end{array}$ & $\begin{array}{l}\text { Local data } \\
(2010-2012) \\
(\%)\end{array}$ & $\begin{array}{l}\text { Present } \\
\text { study } \\
(2016) \\
(\%)\end{array}$ \\
\hline \multirow[t]{2}{*}{ Ciprofloxacin } & MRSA & 96 & 82 & $81 \cdot 3$ \\
\hline & MSSA & 9 & 4 & $3 \cdot 5$ \\
\hline \multirow[t]{2}{*}{ Erythromycin } & MRSA & 82 & 55 & $59 \cdot 5$ \\
\hline & MSSA & 4 & 11 & $15 \cdot 6$ \\
\hline \multirow[t]{2}{*}{ Fusidic acid } & MRSA & $9 \cdot 3$ & 27 & $37 \cdot 5$ \\
\hline & MSSA & $8 \cdot 6$ & 9 & $17 \cdot 5$ \\
\hline \multirow[t]{2}{*}{ Trimethoprim } & MRSA & $20 \cdot 2$ & 24 & $15 \cdot 6$ \\
\hline & MSSA & $3 \cdot 8$ & 16 & $5 \cdot 1$ \\
\hline
\end{tabular}

* MRSA, Methicillin-resistant $S$. aureus; MSSA, methicillin-susceptible $S$. aureus.

isolates referred to the national reference laboratory for epidemiological typing from laboratories in England and Wales in 2002 [11]; the same study reported a PVL prevalence of $4.9 \%$ in isolates associated with skin and soft tissue infections. Other sources have since recorded a higher prevalence of PVL-positive $S$. aureus, notably a 2007 cross-sectional survey of 390 clinical $S$. aureus isolates at a major London hospital with $9 \cdot 7 \%$ of isolates harbouring the gene [31] and with PVL-positivity being four times more frequent in isolates from skin and soft tissue infections than other isolates. A national sentinel surveillance study of community staphylococcal pyogenic skin infections completed by PHE in 2013 found that $36 \%(n=279 / 771)$ cases were PVL-positive [12].

Chlorhexidine is the most frequently used topical antiseptic for skin decolonization in UK healthcare and is an integral part of the control and management of MRSA-positive individuals [7]. Such widespread use may engender resistance and enable certain bacteria to persist in the clinical setting. For a number of reasons, accurate detection of chlorhexidine resistance is technically challenging [17]. These include lack of a consensus on the definition of 'resistance', commonly defined as a raised MIC or MBC $\geqslant 4 \mathrm{mg} / \mathrm{l}$ or the presence of genes known to encode effluxmediated biocide resistance, and lack of an agreed standardized method to assay susceptibility. As a result, the ability of bacteria to withstand chlorhexidine exposure is not routinely tested and possible resistance 
is largely unmonitored. In addition, the relationship between the carriage of chlorhexidine resistance genes and phenotypic reduced susceptibility remains unclear [17].

Our work has shown that there is little evidence of chlorhexidine non-susceptibility in $S$. aureus circulating in the $\mathrm{YH}$ region and there was poor correlation of carriage of $q a c \mathrm{~A}$ with chlorhexidine susceptibility as only eight isolates were positive for this gene. Despite a published method being available to detect $q a c \mathrm{~B}$ and $s m r$ chlorhexidine efflux genes [19], only a qacA-positive control organism could be sourced. In a survey of MRSA bloodstream infection isolates, Otter et al. tested 602 MRSA isolates and identified the qac A gene in $26.2 \%$ isolates; $q a c \mathrm{~B}$ and $s m r$ positive isolates were less frequently identified $(0.3 \%$ and $8 \cdot 1 \%$, respectively) [19]. Although the setting is different (the present study did not just include MRSA isolates) we may infer that screening for $q a c \mathrm{~B}$ and $s m r$ genes may only have identified a small number of additional isolates carrying these genes. It is possible that investigation of the levels of qacA expression in isolates that are phenotypically chlorhexidine susceptible compared with non-susceptible strains may shed further light on this issue. Expression of other genes, such as the regulatory gene, $q a c \mathrm{R}$, or examination of the sequence of the qacA gene itself may help to elucidate if key mutations are necessary for biocide resistance.

We did not collect data about mupirocin/chlorhexidine use/exposure and therefore are not able to draw conclusions about the effects of mupirocin/chlorhexidine on the prevalence of non-susceptibility. As hospital admission records were not recorded, this has restricted any inference regarding co-morbidities and speciality and we cannot estimate the proportion of patients that may have been exposed to chlorhexidine.

$S$. aureus strains circulating within the $\mathrm{YH}$ region are a diverse group and while three lineages predominate $(\mathrm{CC} 30, \mathrm{CC} 15, \mathrm{CC} 1)$, six other lineages are circulating throughout the region. Many of the commonly occurring spa-types identified in the present study are also represented in the 20 most frequent spa-types in MSSA and MRSA isolated in 26 European countries [32]. Notable exceptions include t032 and t021, which map to CC22; t316 and t216, which map to CC59, and $\mathrm{t579}$, which maps to CC398. Such differences in prevalent spa-type may be explained in part by the fact that the European study only included isolates from invasive infections and almost 10 years has passed since the data were collected (September 2006-February 2007).
As genotyping is usually requested following an increase in the number of cases of $S$. aureus (MSSA or MRSA) in a clinical area, the results of the present surveillance provide an important baseline of $S$. aureus circulating in primary care and outside an outbreak situation. In recent years, EMRSA-15 (CC22) and EMRSA-16 (CC30) were the most common MRSA strains in England and Wales; however, while $\mathrm{CC} 22$ remains in circulation, EMRSA-16 is no longer prevalent in the $\mathrm{YH}$ region. Instead MRSA isolates belonging to CC59 have become more predominant.

We acknowledge that there are limitations associated with the present study. The survey sample was designed to minimize systematic bias by capturing all $S$. aureus isolates from all NHS laboratories in the YH region over two consecutive days. The dates of study participation were staggered to ensure that isolates were collected in a timely manner, but each hospital trust was randomized to the dates in order to minimize the potential for over-representation of particular patient groups due to scheduling of specialist clinics or laboratory activity. Prior to isolate collection, it was estimated that the 14 participating laboratories would detect approximately $350 \mathrm{~S}$. aureus isolates per day for patients in both community and hospital settings, hence a 2-day collection period would provide sufficient isolates for precise estimates of mupirocin resistance and chlorhexidine nonsusceptibility prevalence, and PVL toxin-positivity, according to the sample size calculations completed. The target number of samples $(n=700)$ was not reached, resulting in lower levels of precision than expected around the prevalence estimates reported; however, we have tested $>500$ isolates from the general population presenting to a GP or hospital in a large single healthcare region of England. Data have been included from a range of hospital trusts of different size and patient mix. Isolates were drawn from all NHS diagnostic laboratories providing services to the population of YH (5.3 million), covering both rural and urban areas. All observations occurred in the same season but seasonality was not considered likely to impact upon the variables of interest and the risk of seasonality was outweighed by the value of collecting data from all trusts in the same period to permit valid comparison between them.

In summary, the findings of this surveillance suggest that the widespread use of staphylococcal decolonization regimens over the past decade has not had an adverse impact on antimicrobial and biocide resistance 
rates and that mupirocin and chlorhexidine continue to be suitable agents for MRSA decolonization.

\section{ACKNOWLEDGEMENTS}

The authors thank staff in the participating laboratories for collecting the $S$. aureus isolates and completing the datasheets, and Olivia Sherburn, Public Health England, Regional Laboratory, Leeds who completed data entry. We thank Dr Jonathan Edgeworth, Guy's and St Thomas' NHS Trust, London the provision of a control isolate for detection of the qacA gene, and Professor Angela Kearns and colleagues at the Staphylococcus Reference Unit, Public Health England, London for the provision of a control isolate for detection of the mec $\mathrm{C}$ gene, for help and advice with spa-typing and assignment of spa-types to relevant MLST CC and for general discussion about the surveillance project. Thanks to Dr Matthew Wand and Dr Mark Sutton, Public Health England, Porton Down, Salisbury for input and training of chlorhexidine susceptibility testing. Finally, we thank Dr Obaghe Edeghere, Consultant Epidemiologist, Field Epidemiology Service, Public Health England, and Dr Andrew Kirby, Consultant Microbiologist, Leeds Teaching Hospitals NHS Trust, for reviewing the protocol before implementation.

This work was supported by Public Health England.

\section{DECLARATION OF INTEREST}

None.

\section{REFERENCES}

1. European Centre for Disease Prevention and Control. European Antimicrobial Resistance Surveillance System (EARSS-Net) (http://www.rivm.nl/earss/). Accessed 20 May 2016.

2. Hope R, et al. Non-susceptibility trends among staphylococci from bacteraemias in the UK and Ireland, 2001-06. Journal of Antimicrobial Chemotherapy 2008; 62 (Suppl. 2): ii65-ii74.

3. Moreillon P, Que. Y-A, Glauser MP. Staphylococcus aureus (including staphylococcal toxic shock). In: Mandell GL, Bennett JE, Dolin R, eds. Principles and Practice of Infectious Diseases, 6th edn. Philadelphia, USA: Elsevier Churchill Livingstone, 2005, pp. 23212351.

4. Public Health England. Staphylococcus aureus: guidance, data and analysis; (https://www.gov.uk/government/ collections/staphylococcus-aureus-guidance-data-andanalysis\#epidemiology). Accessed 20 May 2016.
5. Popoola VO, et al. Active surveillance cultures and decolonization to reduce Staphylococcus aureus infections in the neonatal intensive care unit. Infection Control and Hospital Epidemiology 2016; 37: 381-387.

6. Schweizer ML, et al. Association of a bundled intervention with surgical site infections among patients undergoing cardiac, hip, or knee surgery. Journal of the American Medical Association 2015; 313: 2162-2171.

7. Health Protection Agency, Association of Medical Microbiologists. Meticillin Resistant Staphylococcus aureus (MRSA) Screening and Suppression. Quick reference guide for Primary Care - for consultation and local adaptation. London, England: Health Protection Agency, 2009.

8. Dinges MM, Orwin PM, Schlievert PM. Exotoxins of Staphylococcus aureus. Clinical Microbiology Reviews 2000; 13: 16-34.

9. Lina G, et al. Involvement of Panton-Valentine leukocidin-producing Staphylococcus aureus in primary skin infections and pneumonia. Clinical Infectious Diseases 1999; 29: 1128-1132.

10. Health Protection Agency. Guidance on the diagnosis and management of PVL-associated Staphylococcus aureus infections (PVL-SA) in England. London, England: Health Protection Agency, 7 November 2008. (https://www.gov.uk/government/uploads/system/ uploads/attachment_data/file/322857/Guidance_on_the_ diagnosis_and_management_of_PVL_associated_SA_ infections_in_England_2_Ed.pdf). Accessed 18 October 2016.

11. Holmes A, et al. Staphylococcus aureus isolates carrying Panton-Valentine leukocidin genes in England and Wales: frequency, characterization, and association with clinical disease. Journal of Clinical Microbiology 2005; 43: 2384-2390.

12. Kearns A, et al. Community staphylococcal pyogenic skin infections in England: PVL, MRSA \& Clonal Diversity. Poster session, presented at the European Congress of Microbiology and Infectious Diseases (ECCMID) 2014 (https://www.escmid.org/escmid publications/escmid_elibrary/?debug=1\%253FtipUrl $\% 253$ Dhttp://\%253A $\% 252 \mathrm{~F} \% 252 \mathrm{Fwww}$.escmid.org $\% 252 \mathrm{~F}$ footnavigation $\% 252 \mathrm{Fdisclaimer} \% 252 \mathrm{~F} \% 253 \mathrm{Fdebug} \% 253$ D1\&tx_solr $\% 5 \mathrm{~B}$ filter $\% 5 \mathrm{D} \% 5 \mathrm{~B} 0 \% 5 \mathrm{D}=$ main_category $\% 253$ ADiagnostic $\% 2$ BBacteriology $\% 2 B \% 2526 \% 2 \mathrm{~B}$ General $\% 2 B M i c r o b i o l o g y \& t x \_s o l r \% 5 B f i l t e r \% 5 D \% 5 B 1$ $\% 5 \mathrm{D}=$ author $\% 253 \mathrm{AAngela} \% 2 \mathrm{BK}$ earns). Accessed 20 September 2016.

13. Ellington MJ, et al. Panton-Valentine leukocidin-related disease in England and Wales. Clinical Microbiologyand Infection 2010; 16: 86-88.

14. Public Health England. UK Standards for Microbiology Investigations: Identification of Staphylococcus species, Micrococcus species and Rothia species. ID 7 Issue 3. London, England: Public Health England, 2014. (https://www.gov.uk/government/uploads/system/uploads/ attachment_data/file/375283/ID_7i3.pdf). Accessed 18 October 2016.

15. European Committee on Antimicrobial Susceptibility Testing. Breakpoint tables for interpretation of 
MICs and zone diameters, 2015. (https://www.gov.uk/ government/uploads/system/uploads/attachment_data/ file/375283/ID_7i3.pdf). Accessed 18 October 2016.

16. Wand ME, et al. Characterization of pre-antibiotic era Klebsiella pneumoniae isolates with respect to antibiotic/disinfectant susceptibility and virulence in Galleria mellonella. Antimicrobial Agents and Chemotherapy 2015; 59: 3966-3972.

17. Horner C, Mawer D, Wilcox M. Reduced susceptibility to chlorhexidine in staphylococci: is it increasing and does it matter? Journal of Antimicrobial Chemotherapy 2012; 67: 2547-2559.

18. Pichon B, et al. Development of a real-time quadruplex PCR assay for simultaneous detection of nuc, PantonValentine leukocidin (PVL), mecA and homologue mec $_{\mathrm{LGA251}}$. Journal of Antimicrobial Chemotherapy 2012; 67: 2338-2341.

19. Otter JA, et al. Selection for qacA carriage in CC22, but not CC30, methicillin-resistant Staphylococcus aureus bloodstream infection isolates during a successful institutional infection control programme. Journal of Antimicrobial Chemotherapy 2013; 68: 992-999.

20. Larsen AR, Stegger M, Sorum M. spa typing directly from a mecA, spa and $p v l$ multiplex PCR assay-a costeffective improvement for methicillin-resistant Staphylococcus aureus surveillance. Clinical Microbiology and Infection 2008; 14: 611-614.

21. Ridom GmbH. Ridom SpaServer (http://www.spaserver. ridom.de/). Accessed 17 January 2016.

22. Garcia-Alvarez L, et al. Meticillin-resistant Staphylococcus aureus with a novel mecA homologue in human and bovine populations in the UK and Denmark: a descriptive study. Lancet Infectious Diseases 2011; 11: 595-603.

23. Hetem DJ, et al. Molecular epidemiology of MRSA in 13 ICUs from eight European countries. Journal of Antimicrobial Chemotherapy 2016; 71: 45-52.
24. Paterson GK, et al. Prevalence and characterization of human mecC methicillin-resistant Staphylococcus aureus isolates in England. Journal of Antimicrobial Chemotherapy 2014; 69: 907-910.

25. Petersen A, et al. Epidemiology of methicillin-resistant Staphylococcus aureus carrying the novel mec $\mathrm{C}$ gene in Denmark corroborates a zoonotic reservoir with transmission to humans. Clinical Microbiology and Infection 2013; 19: E16-E22.

26. Vivoni AM, et al. Mupirocin for controlling methicillinresistant Staphylococcus aureus: lessons from a decade of use at a university hospital. Infection Control and Hospital Epidemiology 2005; 26:662-667.

27. Simor AE, et al. Mupirocin-resistant, methicillin-resistant Staphylococcus aureus strains in Canadian hospitals. Antimicrobial Agents and Chemotherapy 2007; 51: 3880 3886.

28. Nagant C, et al. Low prevalence of mupirocin resistance in Belgian Staphylococcus aureus isolates collected during a 10 year nationwide surveillance. Journal of Antimicrobial Chemotherapy 2016; 71: 266-267.

29. Fawley WN, et al. Surveillance for mupirocin resistance following introduction of routine peri-operative prophylaxis with nasal mupirocin. Journal of Hospital Infection 2006; 62: 327-332.

30. Horner C, et al. Meticillin-resistant Staphylococcus aureus in elderly residents of care homes: colonization rates and molecular epidemiology. Journal of Hospital Infection 2013; 83: 212-218.

31. Shallcross LJ, et al. Panton-Valentine leukocidin associated staphylococcal disease: a cross-sectional study at a London hospital, England. Clinical Microbiologyand Infection 2010; 16: 1644-1648.

32. Grundmann H, et al. Geographic distribution of Staphylococcus aureus causing invasive infections in Europe: a molecular-epidemiological analysis. PLoS Medicine 2010; 7: e1000215. 